\title{
Physical health of autistic girls and women: a scoping review
}

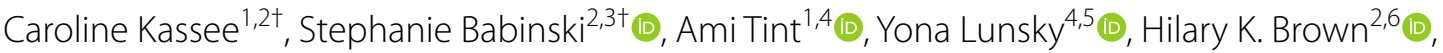 \\ Stephanie H. Ameis ${ }^{1,4,5,7}$ (D) Peter Szatmari ${ }^{1,5,7}$, Meng-Chuan Lai ${ }^{1,4,5,7,8,9,10^{*+}}$ (D) and Gillian Einstein ${ }^{2,8,11,12+}$ (1)
}

\begin{abstract}
Background: There is a growing recognition of sex and gender influences in autism. Increasingly, studies include comparisons between sexes or genders, but few have focused on clarifying the characteristics of autistic girls'/women's physical health.
\end{abstract}

Methods: A scoping review was conducted to determine what is currently known about the physical health of autistic girls/women. We screened 1112 unique articles, with 40 studies meeting the inclusion criteria. We used a convergent iterative process to synthesize this content into broad thematic areas.

Results: Autistic girls/women experience more overall physical health challenges compared to non-autistic girls/ women and to autistic boys/men. Emerging evidence suggests increased prevalence of epilepsy in autistic girls/ women compared to non-autistic girls/women and to autistic boys/men. The literature also suggests increased endocrine and reproductive health conditions in autistic girls/women compared to non-autistic girls/women. Findings regarding gastrointestinal, metabolic, nutritional, and immune-related conditions are preliminary and inconsistent.

Limitations: The literature has substantial heterogeneity in how physical health conditions were assessed and reported. Further, our explicit focus on physical health may have constrained the ability to examine interactions between mental and physical health. The widely differing research aims and methodologies make it difficult to reach definitive conclusions. Nevertheless, in keeping with the goals of a scoping review, we were able to identify key themes to guide future research.

Conclusions: The emerging literature suggests that autistic girls/women have heightened rates of physical health challenges compared to non-autistic girls/women and to autistic boys/men. Clinicians should seek to provide holistic care that includes a focus on physical health and develop a women's health lens when providing clinical care to autistic girls/women.

Keywords: Autism, Physical health, Sex differences, Gender, Girls, Women, Scoping review

*Correspondence: mengchuan.lai@utoronto.ca

${ }^{\dagger}$ Caroline Kassee and Stephanie Babinski are equal contribution joint first authors

${ }^{\dagger}$ Meng-Chuan Lai and Gillian Einstein are equal contribution joint senior authors

${ }^{1}$ The Margaret and Wallace McCain Centre for Child, Youth and Family Mental Health, Centre for Addiction and Mental Health, 80 Workman Way, Toronto, ON M6J 1H4, Canada

Full list of author information is available at the end of the article

\section{Background}

Autism spectrum disorder (hereafter autism) is a neurodevelopmental condition characterized by early-onset social communication difficulties and repetitive, stereotyped behaviors. The estimated prevalence rate of autism is approximately $1 \%$ worldwide [1], with a higher prevalence among males than females [2,3]. The widely reported male-to-female ratio for autism prevalence is 4-5:1 but large-scale, population-based epidemiological

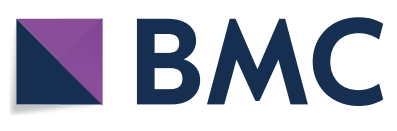

(c) The Author(s) 2020. Open Access This article is licensed under a Creative Commons Attribution 4.0 International License, which permits use, sharing, adaptation, distribution and reproduction in any medium or format, as long as you give appropriate credit to the original author(s) and the source, provide a link to the Creative Commons licence, and indicate if changes were made. The images or other third party material in this article are included in the article's Creative Commons licence, unless indicated otherwise in a credit line to the material. If material is not included in the article's Creative Commons licence and your intended use is not permitted by statutory regulation or exceeds the permitted use, you will need to obtain permission directly from the copyright holder. To view a copy of this licence, visit http://creativecommons.org/licenses/by/4.0/. The Creative Commons Public Domain Dedication waiver (http://creativeco mmons.org/publicdomain/zero/1.0/) applies to the data made available in this article, unless otherwise stated in a credit line to the data. 
studies suggest that the ratio is in fact lower, at 3-4:1 [4]. The higher rates of autism among males reflect sex and gender differences in the likelihood of developing autism and potential gender biases in clinical assessment and diagnoses $[5,6]$.

Autism is highly associated with co-occurring health conditions [7]. It is hypothesized that this likely reflects complex epigenetic and pleiotropic gene-environment interactions and behavioral mechanisms [3, 8], which complicate the clinical presentation of autism. Co-occurring health conditions in autism are associated with varied developmental trajectories $[9,10]$ and unique social and psychological challenges experienced throughout the life course [3]. In order to improve our knowledge of sexbased and gender-based differences in autism [11], it is critical to better understand the prevalence and characteristics of co-occurring conditions in autistic girls and women.

Most research on co-occurring conditions in autistic girls/women relative to boys/men has focused on psychiatric conditions, suggesting increased internalizing psychopathology in autistic girls/women compared to in boys/men [12-14]. The latest meta-analysis on cooccurring psychiatric diagnoses in autistic people shows that studies with a higher proportion of girls/women tend to find higher rates of depression [15]. However, less attention has been paid to sex and gender differences in autism outside of the domain of mental health, especially regarding physical health. (Note that we define physical health in this context to encompass non-mental health conditions within the broad category of medical disorders or problems.) Accurate and in-depth information in this domain, especially concerning autistic girls/ women, is essential to the provision of comprehensive and sex- and gender-sensitive health care and is important for elucidating clinically useful subgroups within the autism spectrum. In view of this, we conducted a scoping review of the literature, focused on the extent and range of research pertaining to the physical health of autistic girls/women. Two research questions guided our review: (1) What do we know about the physical health of autistic girls/women; and (2) How specific are these physical health concerns to autistic girls/women, as compared to autistic boys/men, and to non-autistic girls/women?

\section{Methods}

We conducted a scoping review of the literature following the methodological framework outlined by Arksey and O'Malley [16] and recent Preferred Reporting Items for Systematic Reviews and Meta-analyses standards for scoping reviews (PRISMA-ScR) [17]. Scoping reviews allow a broad survey of the literature in a particular area to determine existing themes and areas of inquiry that are under-researched. Scoping reviews typically do not conduct an assessment of bias in the research nor do they appraise or generate effect sizes [16]. We considered a scoping review to be the most appropriate approach for examining emerging evidence concerning the physical health of autistic girls/women since it was unclear what specific questions should be posed in this area given the limitations of current literature. Therefore, our purposes were to summarize the extent and range of research pertaining to physical health in autistic girls/women and to identify evidence gaps. In this way, we surveyed all of the literature with respect to physical health in autistic girls/women, without restrictions based on study design or comparison groups. Furthermore, as this literature often-unfortunately-conflates gender and sex, it is difficult to tease apart their respective effects. Hence, in interpreting the findings, references to "girls/women" were assumed to refer to biological, cis-gender females and references to "gender" were read very carefully to determine whether they referred to biological sex or gender identity.

We systematically searched the following databases according to PRISMA standards [18]: CINAHL, PubMed, EMBASE, PsycINFO, Scopus, and Web of Science (see Additional file 1: Appendix: Search Strategy). As this was a scoping review aimed at assessing general themes in the published literature rather than analyzing specific types of data, grey literature was not included in the searches. Autism and co-occurring physical health conditions were defined using a combination of keywords and controlled vocabulary applicable to each database (see Additional file 1: Appendix: Search Strategy). We purposely kept the definition of "physical health" as broad as possible, in order to gather a wide range of studies and gain a thorough coverage of the published literature with respect to non-mental health-related conditions. There was no publication type or date restriction at this stage, but the search results were limited to human studies and journal articles written in English. The final database search was performed on December 5, 2019, and references were managed using Mendeley (https://www.mendeley.com/).

A systematic selection process was used to determine the final articles included in this review. After duplicates were removed, two authors (CK and SB) screened titles and abstracts with support from senior authors (M-CL and GE), using broad criteria to allow for the inclusion of any potentially relevant study for further evaluation. Fulltext articles were evaluated for inclusion by CK and SB. The pool of studies identified based on screening titles, abstracts, and consultations with senior authors determined the inclusion and exclusion criteria. At this stage, articles were included if they: (1) reported on co-occurring physical health conditions in people with a diagnosis 
of autism as defined by the DSM-IV, DSM-5, or ICD-10 criteria, or had direct relevance to the physical health of autistic girls/women; (2) included a clearly articulated sex-specific or gender-specific description or analysis of these conditions; (3) studied biological females only, or if the total female autism sample size was $\geq 15$ and with at least one-eighth (12.5\%) of the total autism sample being biologically female (to ensure that included studies had a sufficient number of girls/women to derive sex-specific or gender-specific information); (4) reported original, English-language research articles or reviews published in peer-reviewed scientific journals; and (5) in the case of review articles, used systematic search methods and included sex-specific or gender-specific analyses and interpretation. Articles were excluded if they were: (1) review articles using non-systematic search methodology; (2) opinion pieces; (3) editorials; (4) case reports; or (5) conference papers. Final decisions on which articles to include were made in discussion within the research team. Articles were grouped by main topic area and study design for organizational clarity. Data were extracted as shown in Tables 1 and 2, with relevant findings summarized in the Results section. We used a convergent iterative process involving multistage revisions among all authors to synthesize included studies into a series of themes that broadly summarize key findings in the literature.

\section{Results}

\section{Search results}

We screened a total of 1112 unique citations and reviewed the full text of 201 articles, with 40 studies ultimately meeting the inclusion criteria (Fig. 1). The majority of the studies were from North America and Europe/ UK, cross-sectional, and about general prevalence rates for health conditions in autistic individuals (Table 1). The articles included descriptive studies of autistic girls/ women only, as well as studies of autistic individuals of all ages and functional levels that compared autistic girls/ women to autistic boys/men and/or non-autistic girls/ women (Table 2).
Three major themes on the physical health status of autistic girls/women emerged from the existing literature: (1) Autistic girls/women experience more overall physical health conditions than autistic boys/men and non-autistic girls/women; (2) Epilepsy has been most commonly researched and seems more common in autistic girls/ women compared to autistic boys/men and non-autistic girls/women; and (3) Autistic girls/women may experience more menstruation-related complications, endocrine and reproductive health conditions compared to non-autistic girls/women. Finally, studies that met the inclusion criteria but that did not align with the three major themes are described in a fourth section, Miscellaneous Emerging Findings. Details of sample characteristics, methodology, and statistics are provided in Table 2.

\section{Summary of key themes}

Theme 1: Overall Physical Health Status

Thirteen out of the 40 included studies focused on the overall physical health status of autistic girls/women [19-31], with respect to multiple conditions across body systems.

Autistic girls/women compared to autistic boys/men Six studies explored the prevalence of various co-occurring physical health conditions in autistic girls/women and autistic boys/men [19-24]. Two of these six studies focused on population-based registry data: one in autistic children and youth (25,063 individuals with autism) [19] and the other in autistic adults (6649 with autism) [20]. Both reported higher odds for deafness, blindness, and physical disability in autistic girls/women compared to autistic boys/men $[19,20]$. A third study [21], a followup on the same adult cohort in [20], found that autistic women in the 25-34 years of age range were more likely to report "poor general health" compared to autistic men [21]. Two additional studies reported on prevalence of physical health conditions as a percentage of their total registry-based autism sample [22, 23]. In 4123 autistic youth in a clinical registry, most physical health conditions were more common in autistic girls/women than in autistic boys/men (e.g., allergy/immunologic conditions,

Table 1 Overview of included studies $(n=40)$

\begin{tabular}{lll}
\hline Years of publication & Country of origin & Study design \\
\hline $2007-2020$ & North America $=17$ & Systematic reviews and meta-analyses $=5$ \\
& Europe and UK $=12$ & Reviews with systematic search methods $=2$ \\
& Asia $=5$ & Cross-sectional studies, with population $/$ registry samples $=20$ \\
& Middle East $=3$ & Cross-sectional studies, with clinic/community samples $=13$ \\
& Australia $=3$ & \\
Africa $=0$ & \\
South America $=0$ & \\
\hline
\end{tabular}




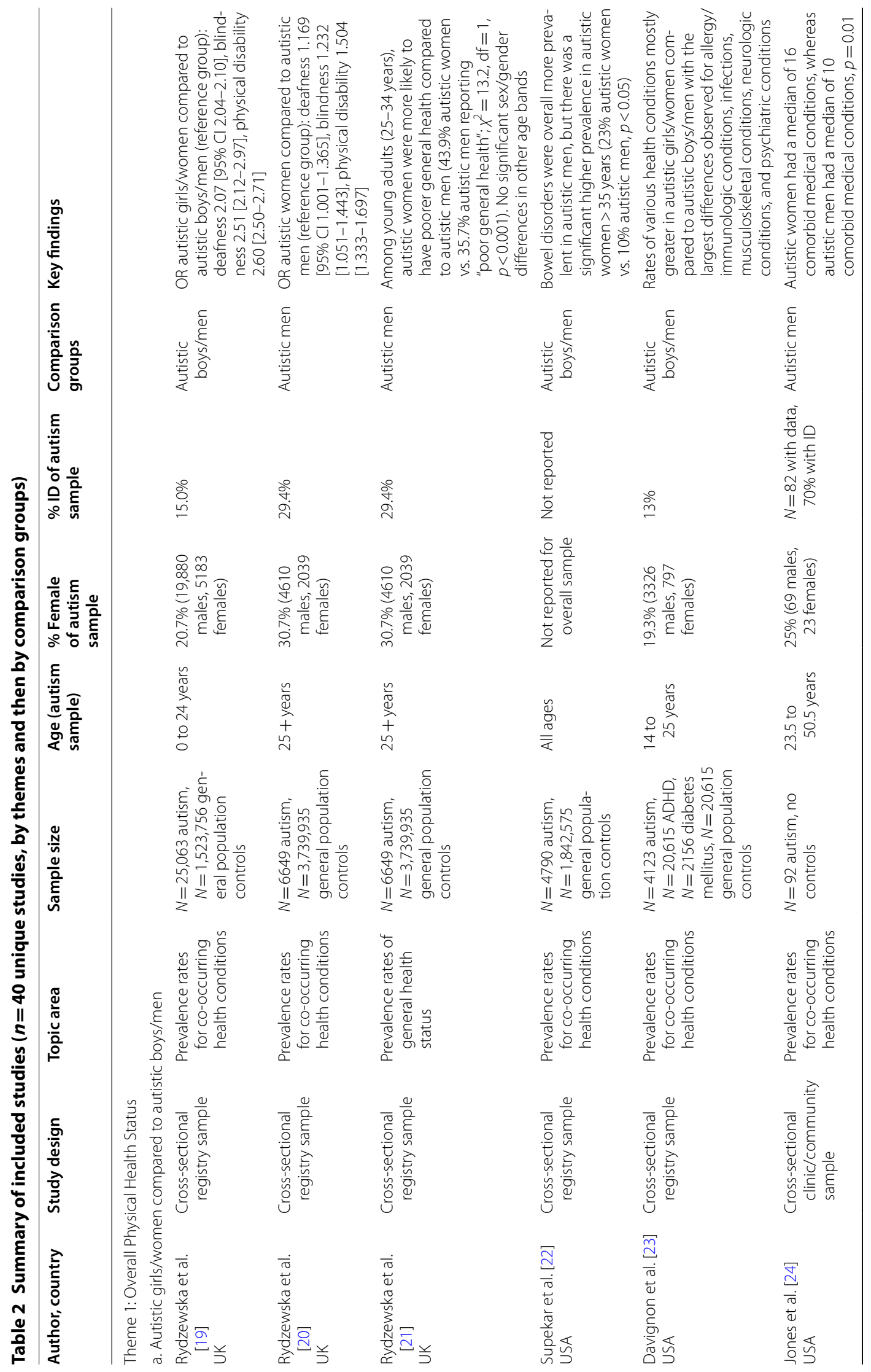




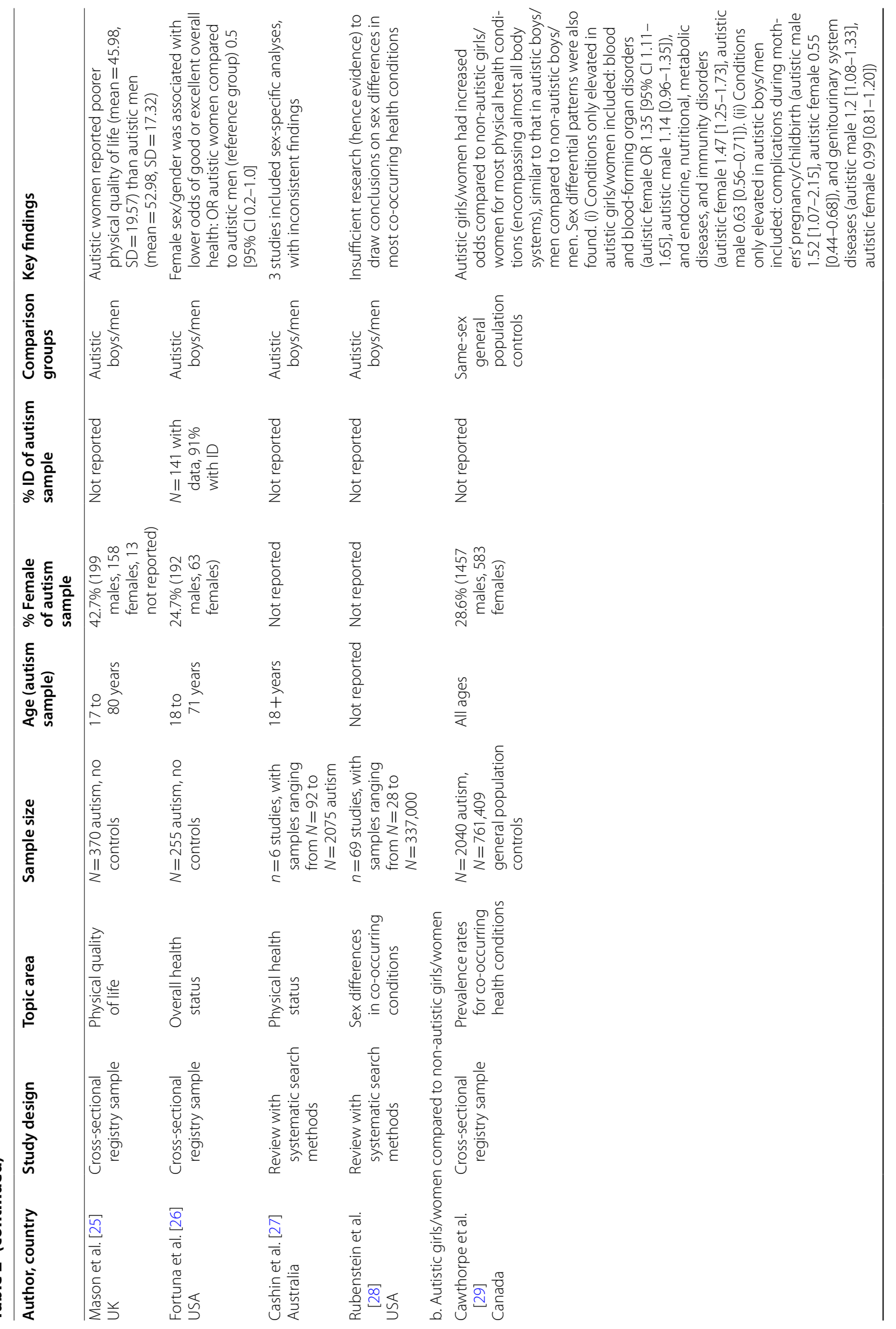




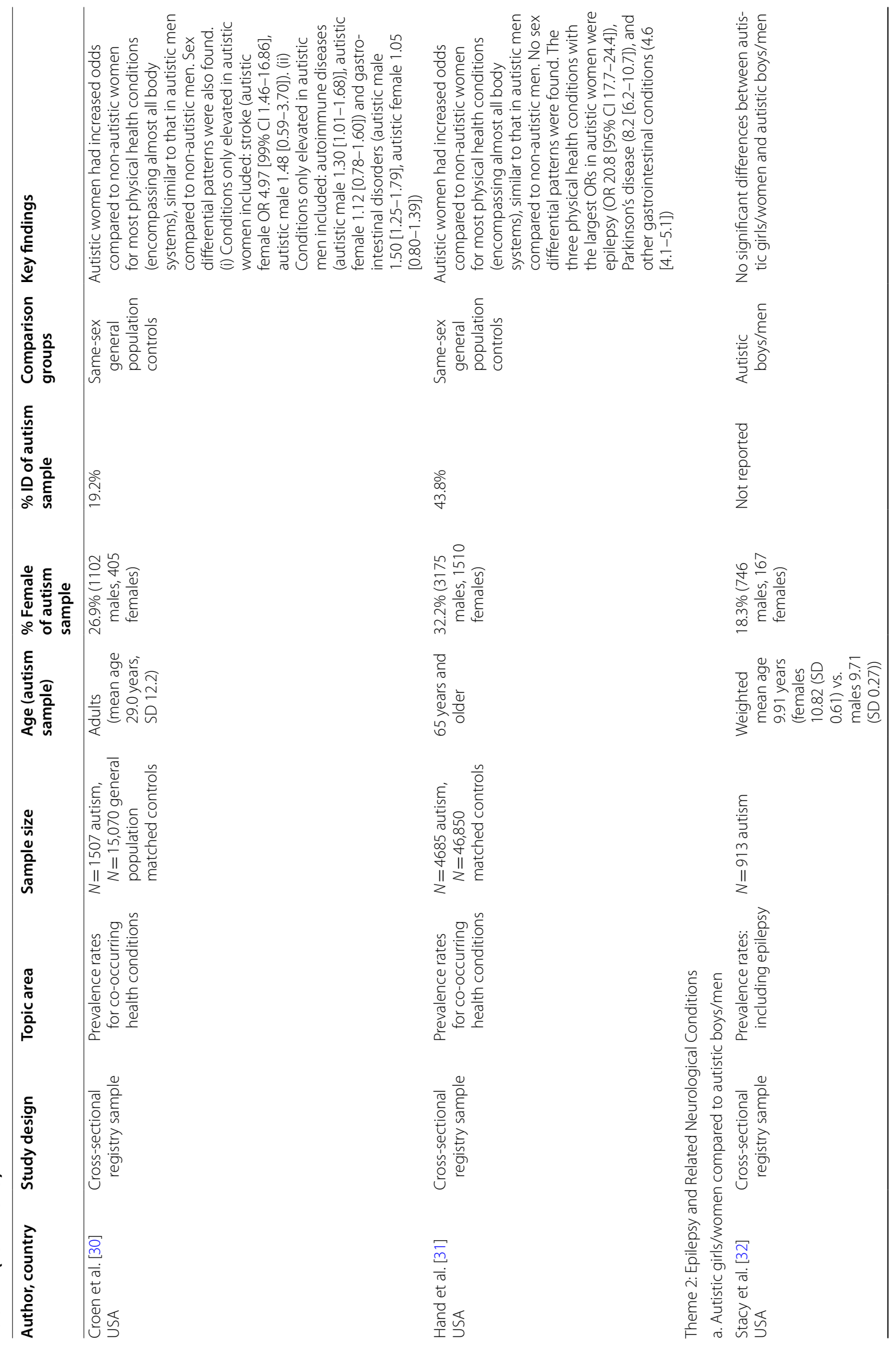




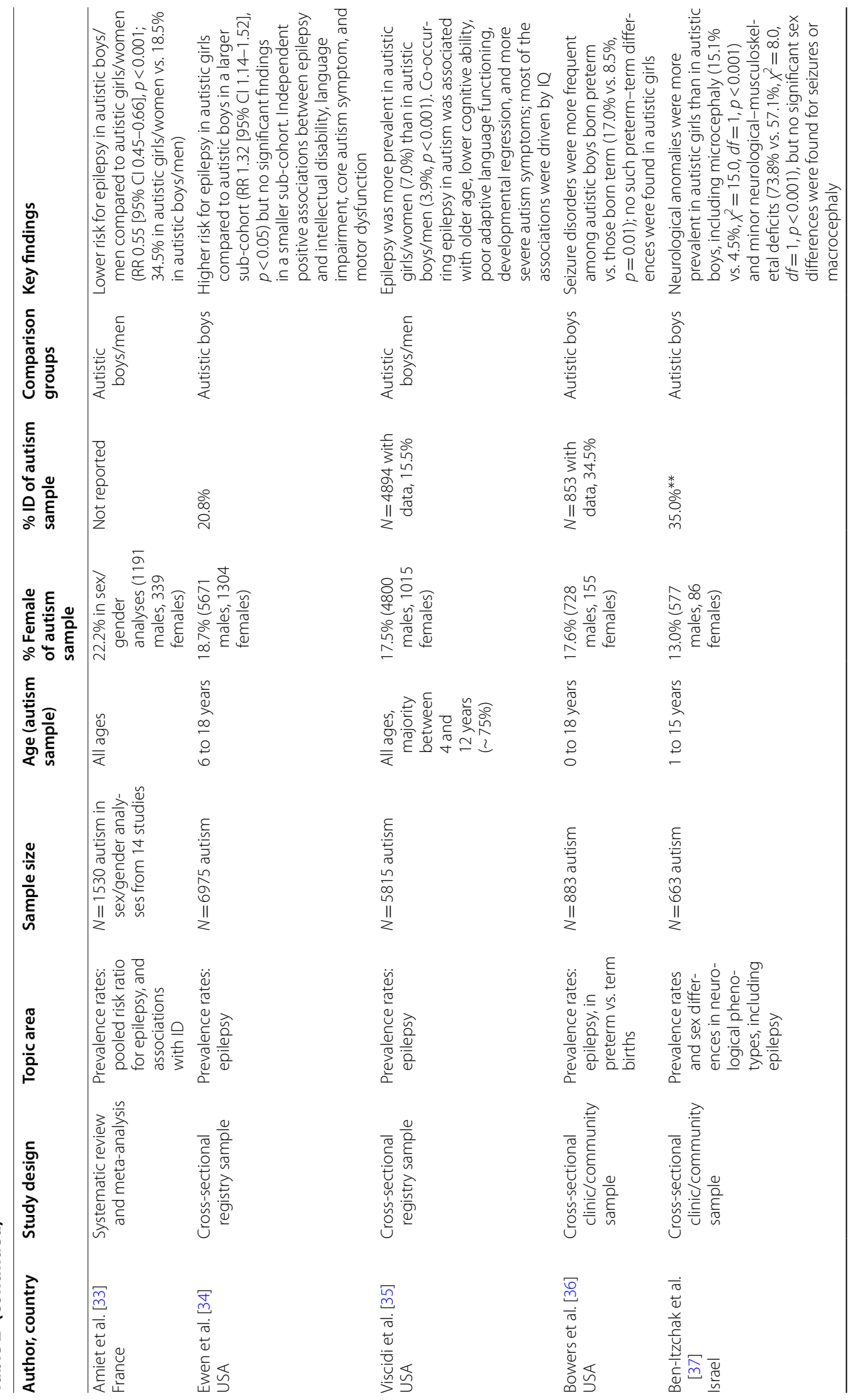




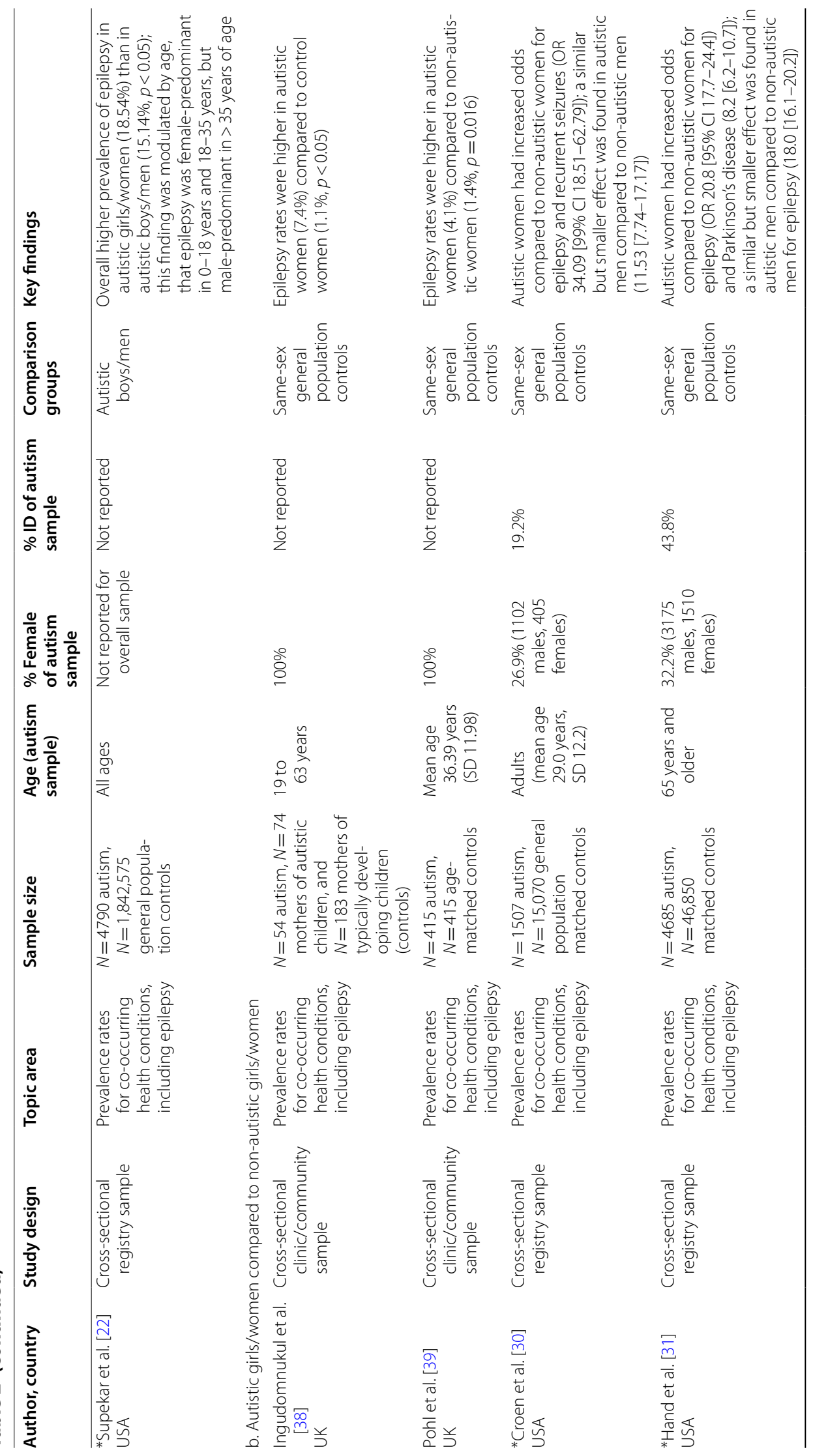




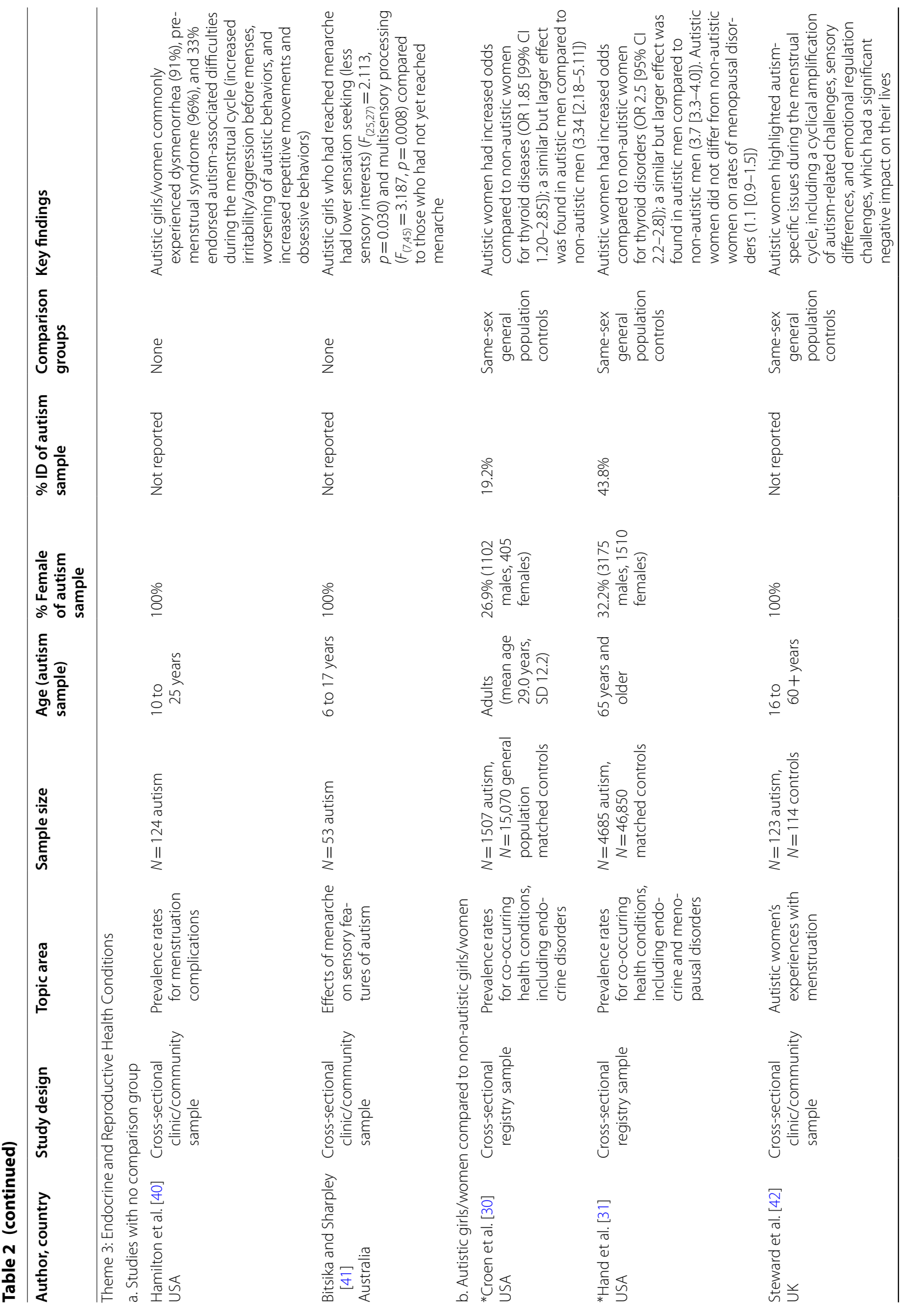




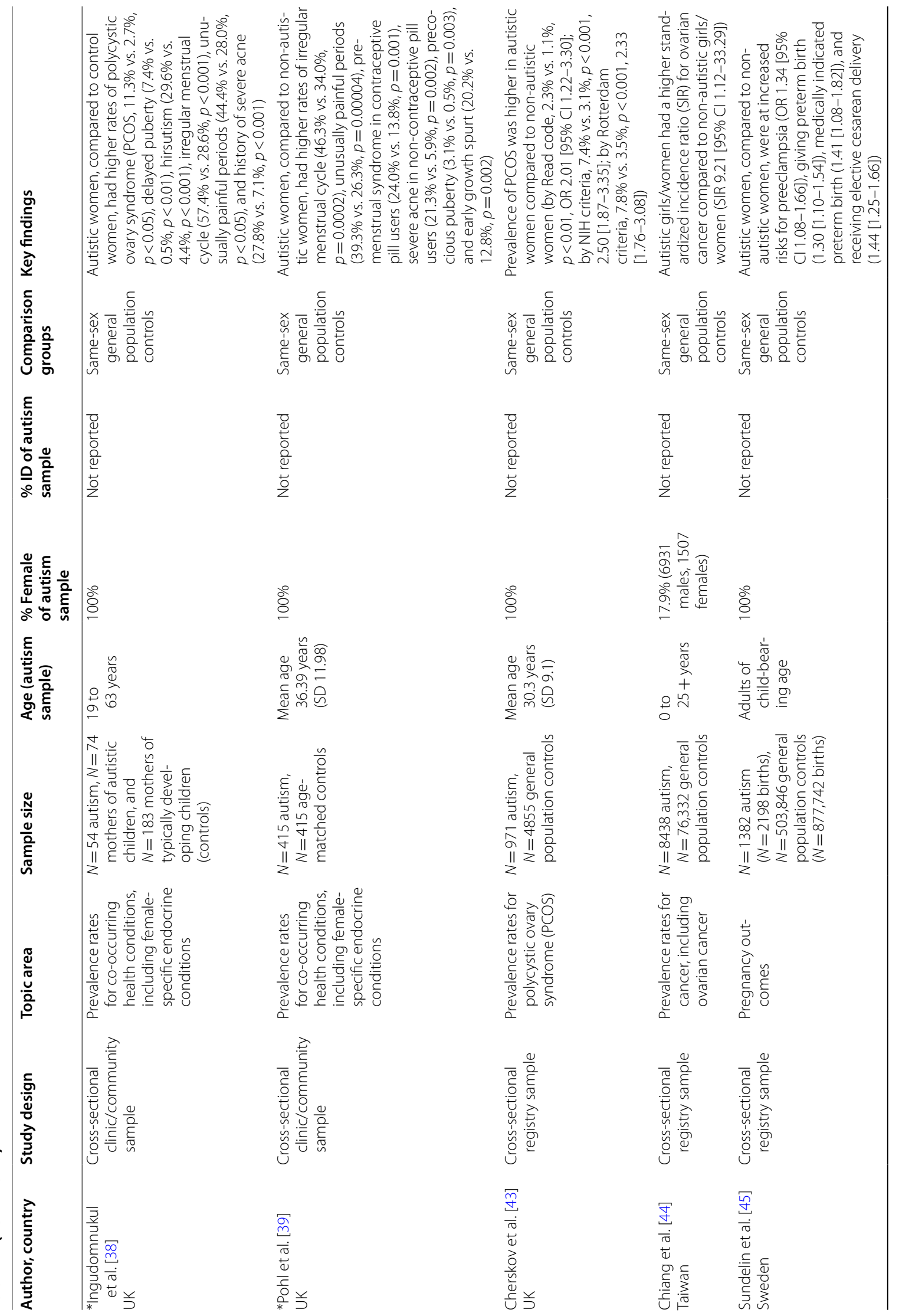




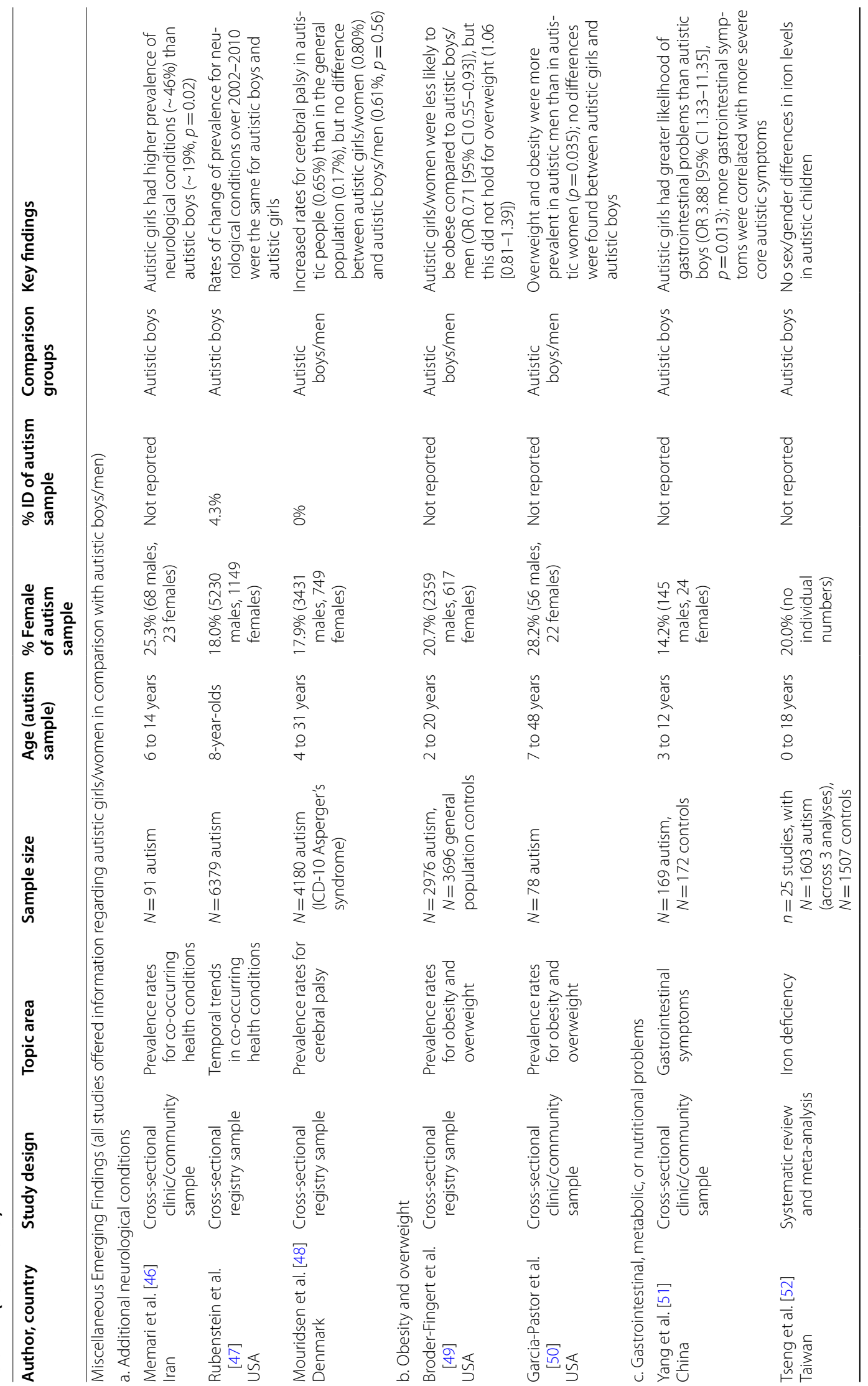




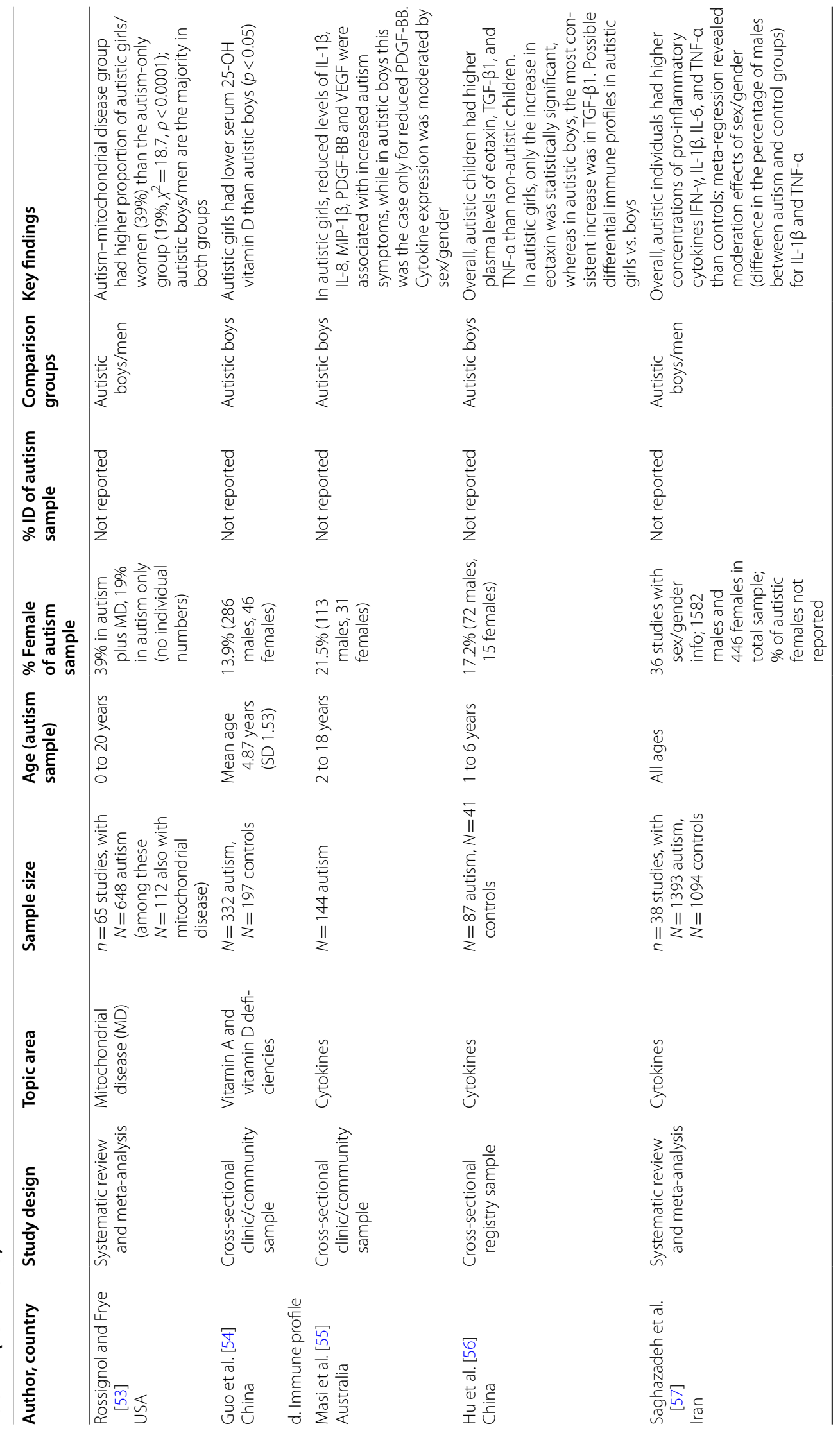




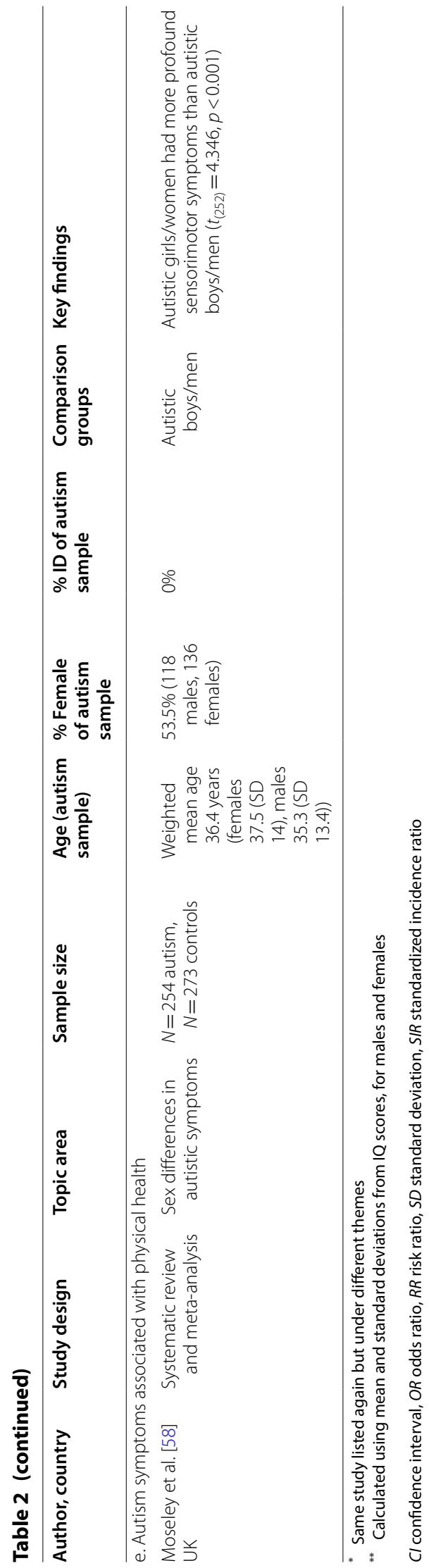




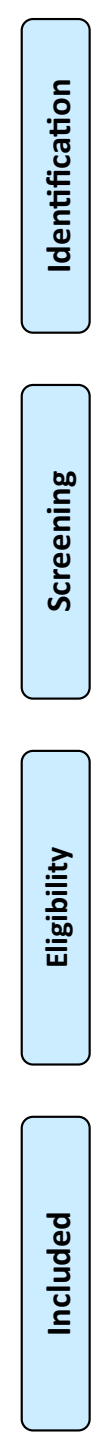

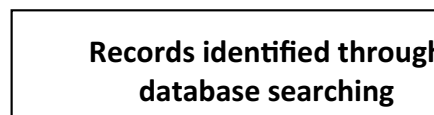
$(n=2,543)$
Additional records identified through other sources $(n=0)$

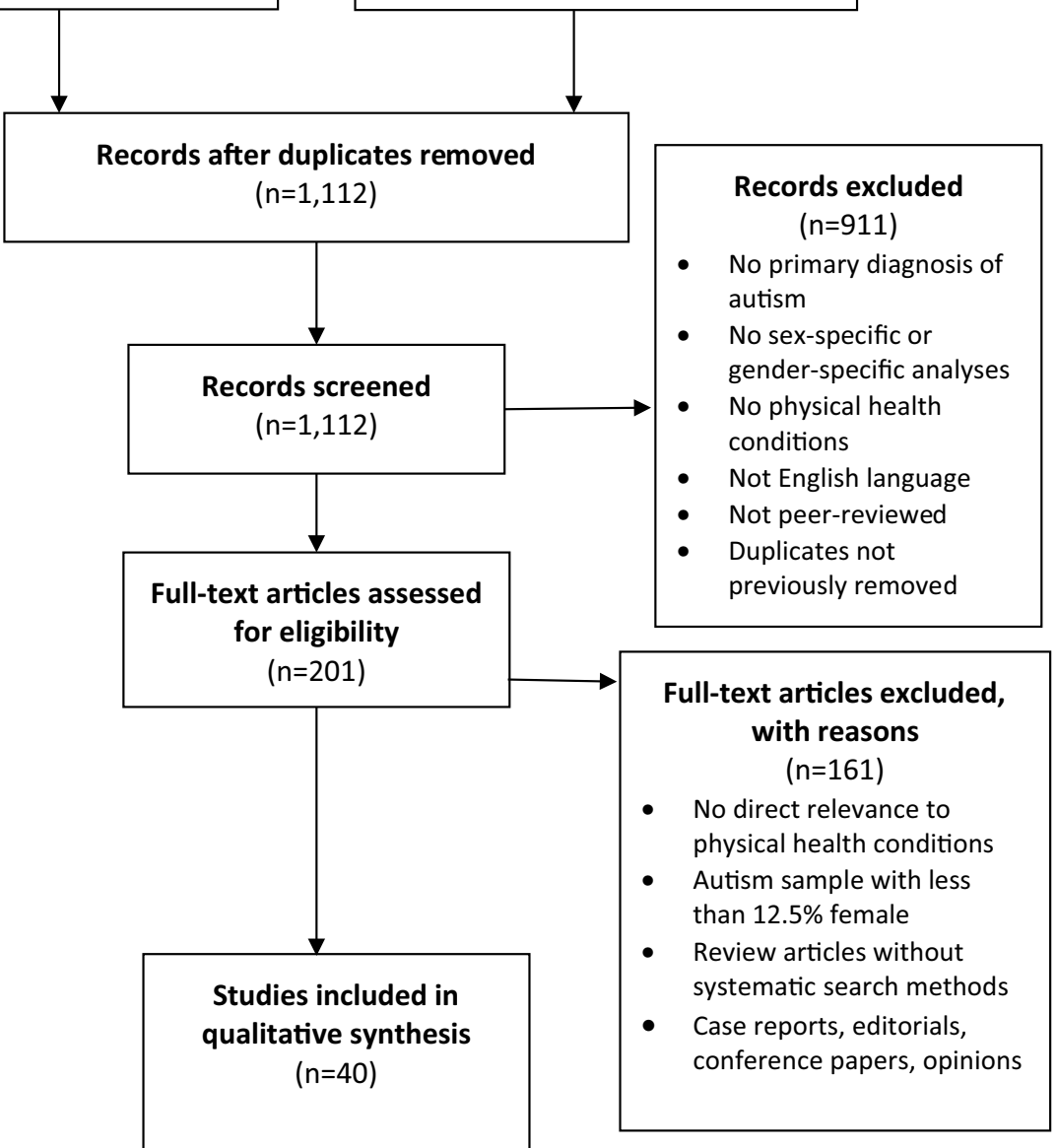

Fig. 1 PRISMA flow diagram for study selection

infections, musculoskeletal conditions, neurologic conditions) [23]. In 4790 autistic people of all ages from large medical registry cohorts, there was a higher prevalence of bowel disorders in autistic women compared to autistic men $>35$ years of age [22]. The sixth study found a higher median number of medical comorbidities in autistic women compared to autistic men, in a community sample of 92 autistic adults [24].

Two studies further examined sex/gender as a predictor of physical quality of life and overall health status in 370 [25] and 255 [26] autistic adults. Female sex/gender was a significant predictor of lower physical healthrelated quality of life [25] and lower overall health [26]. Finally, two literature reviews on the physical health status of autistic adults regardless of sex/gender [27] and sex differences in developmental and medical endophenotypes in autism [28], respectively, reported there was insufficient research to reach consensus about sex/gender differences.

Autistic girls/women compared to non-autistic girls/ women Three studies with population-based registry samples examined the overall physical health status of autistic girls/women compared to non-autistic girls/ women [29-31]. Two of them compared autistic adults to same-sex matched controls (2040 autistic individuals vs. 761,409 general population individuals [29] and 1507 autistic individuals vs. 15,070 general population controls 
[30]), and the other exclusively studied autistic seniors ( $\geq 65$ years of age) who were enrolled in US-based Medicare (4685 with autism vs. 46,850 controls [31]). All three studies found elevated odds for most physical health conditions in autistic girls/women compared to non-autistic girls/women [29-31]. As a side note (also corresponding to the previous section), two of them also described odds ratios (ORs) of physical health conditions in autistic girls/ women (reference to non-autistic girls/women) in contrast to autistic boys/men (reference to non-autistic boys/ men) $[29,30]$ : Some conditions showed elevated ORs only in autistic girls/women, whereas others showed elevated ORs only in autistic boys/men (Table 2), alongside the majority of other conditions showing sex-similar patterns of elevated ORs across autistic individuals [29-31].

\section{Theme 2: Epilepsy and Related Neurological Conditions}

Eleven out of the 40 included studies specifically focused on, or included, epilepsy when examining sex/gender differences in neurological conditions in autistic individuals [22, 30-39].

Autistic girls/women compared to autistic boys/men Five studies compared autistic girls/women to autistic boys/ men with respect to epilepsy [22,32-35]. While one study reported no sex differences in ORs for epilepsy [32], the other four studies found elevated rates of epilepsy in autistic girls/women compared to autistic boys/men. However, this finding was also associated with age, cognitive ability, adaptive functioning, language ability, and autism symptom severity [22, 33-35]. One meta-analysis with pooled sample sizes of 1530 autistic children and adults from 14 studies [33], and another study with 6975 autistic children from registry-based cohorts [34], found higher rates of epilepsy in autistic girls/women compared to autistic boys/men. They also found positive associations between rates of epilepsy and intellectual disability [33, 34], language impairment, core autism symptoms, and motor dysfunction [34]. Two other registry-based studies on prevalence rates for comorbid health conditions, including epilepsy, in 5815 [35] and 4790 [22] autistic individuals found higher prevalence of epilepsy in autistic girls/ women compared to autistic boys/men. The sex difference patterns were modulated by age $[22,35]$, cognitive ability, adaptive language functioning, developmental regression, and autism symptom severity [35].

In addition, two studies specifically explored neurological profiles including seizure disorders in autistic children from community-based samples [36, 37]. One study of 883 autistic children found seizure disorders to be more frequent among autistic boys born preterm vs. those born term, while no preterm-term differences were found in autistic girls [36]. The other study on 663 autistic children found higher prevalence of neurological anomalies (e.g., microcephaly, minor neurological-musculoskeletal deficits) in autistic girls than in autistic boys, but no sex differences in seizures [37].

Autistic girls/women compared to non-autistic girls/ women Four studies reported risks of epilepsy in autistic girls/women against non-autistic girls/women [30, $31,38,39$ ]. A population-based registry study of autistic adults found that autistic women were at significantly higher risks of having epilepsy than non-autistic women [30]. In another registry study on physical health in autistic seniors, epilepsy shows the largest OR among all conditions in autistic women compared to non-autistic women [31]. Similarly, two additional community-based studies reported elevated rates of epilepsy in autistic women compared to non-autistic women in a sample of 54 autistic women and 183 non-autistic women [38] and in another sample of 415 autistic women and 415 nonautistic women [39].

\section{Theme 3: Endocrine and Reproductive Health Conditions}

Ten out of the 40 included studies focused on endocrine and reproductive health conditions in autistic girls/ women only $[40,41]$ or in comparison with non-autistic girls/women [30, 31, 38, 39, 42-45].

Studies with no comparison group Two communitybased studies reported menstruation-related health challenges in autistic girls/women [40, 41]. One online survey with 124 autistic girls/women found $>90 \%$ of them experienced dysmenorrhea and premenstrual syndrome (PMS), while one-third endorsed increased autism-associated difficulties during the menstrual cycle [40]. The other study on the effects of menarche on the sensory features of autism in 53 autistic girls reported that those who reached menarche had lower sensory interests and multisensory processing than those who had not yet reached menarche [41].

Autistic girls/women compared to non-autistic girls/ women Eight studies examined endocrine and reproductive health conditions in autistic girls/women compared to non-autistic girls/women [30, 31, 38, 39, 42-45]. A population-based registry study found that autistic women were at higher risks of having thyroid diseases than non-autistic women [30]. Another registry study on physical health in autistic seniors yielded similar findings regarding thyroid diseases, but autistic women had a comparable rate of menopausal disorders compared to non-autistic women [31]. A community-based qualitative study on 123 autistic and 114 non-autistic women's experiences of menstruation found that, despite many overlap- 
ping challenges reported by both groups, autistic women highlighted amplification of autism-related (e.g., sensory and emotional regulation) challenges in sync with the menstrual cycle, which had significant negative impact on their lives [42]. Three studies focused on the prevalence of female-specific endocrine conditions: one from 54 autistic women and 183 non-autistic women [38] and the other from 415 autistic women and 415 non-autistic women [39] recruited from the community, as well as another registry-based study on nation-wide electronic health records of 971 autistic women and 4855 non-autistic women [43]. The findings demonstrated higher rates of irregular menstrual cycle [38, 39], unusually painful periods $[38,39]$, and polycystic ovary syndrome (PCOS) $[38$, 43 ] in autistic women compared to non-autistic women, along with a range of other endocrine-related conditions [38, 39]. Furthermore, a nation-wide registries study of cancer risks with 8438 autistic children, youth, and young adults compared to 76,332 general population controls found a higher incidence of ovarian cancer in autistic girls/women compared to non-autistic girls/women [44]. Finally, a registry-based study on pregnancy outcomes in 2198 births to 1382 autistic women of child-bearing age, compared to 877,742 births in 503,846 women from the general population, found increased risk of preeclampsia, giving preterm birth, and receiving elective cesarean delivery for autistic women compared to non-autistic women [45].

\section{Miscellaneous emerging findings}

Thirteen out of the 40 studies [46-58] were in emerging areas of research that did not align well with the three major themes; they also provided relatively inconsistent findings. All 13 studies offered information regarding autistic girls/women in comparison with autistic boys/ men.

\section{Additional neurological conditions}

Three studies reported on neurological conditions beyond epilepsy in autism [46-48], with two examining neurological conditions as a broad category [46, 47] and the third, cerebral palsy [48]. One community-based study of 91 autistic children found that autistic girls had higher rates of neurological conditions than autistic boys [46]. A surveillance registry-based study found that in 6379 8-year-old autistic children across eight US sites, both autistic girls and boys showed stable rates of change in the prevalence of neurological conditions over 2002-2010 [47]. The final study, in a nation-wide cohort of 4180 autistic individuals (with ICD-10 Asperger's syndrome), found increased prevalence of cerebral palsy in autistic individuals compared to the general population, without significant differences between autistic girls/ women and boys/men [48].

\section{Obesity and overweight}

Two studies focused on obesity and overweight (via body mass index) $[49,50]$. One registry-based study including 2976 autistic children and youth found that autistic girls were less likely to be obese compared to autistic boys, but they were equally likely to be overweight [49]. The other study, a community-based study of 78 autistic children, adolescents and adults, found that being overweight, as well as being obese, was more common in autistic men than in autistic women but with no differences found between autistic boys and autistic girls [50].

\section{Gastrointestinal, metabolic, or nutritional problems}

Four studies focused on gastrointestinal, metabolic, or nutritional conditions, comparing autistic girls/women and autistic boys/men [51-54]. One community-based study examined the prevalence of gastrointestinal problems in 169 autistic children, reporting that autistic girls had a greater likelihood of gastrointestinal problems than autistic boys and that more gastrointestinal symptoms were correlated with more severe core autistic symptoms [51]. Two were systematic reviews and meta-analyses: One, on peripheral iron levels and iron deficiency (1603 autistic children pooled from 25 studies across 3 analyses), found no sex/gender differences with respect to iron levels in autistic children [52]; the other, on mitochondrial dysfunction (648 autistic children and youth pooled from 65 studies), found a higher proportion of girls/ women in the group of those with autism plus mitochondrial disease compared to those with autism only [53]. The final study, a community-based study on vitamins A and $\mathrm{D}$ deficiencies in 332 autistic children, reported that autistic girls had lower serum 25-OH vitamin D than autistic boys [54].

\section{Immune profile}

Three studies focused on sex-specific immunological features in autism [55-57]. Cytokine levels were measured in two studies of autistic children and adolescents from registry- and hospital-based cohorts: one with 144 (27 cytokines measured) [55] and the other, 87 (11 cytokines measured) [56] autistic individuals. Both studies found differences in the cytokine profiles of autistic girls compared to autistic boys but on different cytokines $[55,56]$. A meta-analysis of 38 studies on 1393 autistic individuals regarding circulating pro-inflammatory cytokines also found sex/gender differences in cytokine patterns [57]. Altogether, these preliminary findings implicate potential 
sex/gender differences in immune profiles related to autism, though with inconsistent cytokine involvement.

\section{Autism symptoms associated with physical health}

Lastly, one meta-analysis examined sex/gender differences in 254 autistic adults regarding self-reported autistic characteristics, which included sensorimotor symptoms, some of which related to physical health, such as sensitivity to pain. Results revealed more severe sensorimotor symptoms in autistic women than in autistic men [58].

\section{Discussion}

The purpose of this scoping review was to explore what is known about co-occurring physical health conditions in autistic girls/women. Out of the 201 full-text articles we reviewed, only 40 met our inclusion criteria, mainly due to the paucity of reporting on sex or gender differences among populations with autism and the low percentages of autistic girls/women included in the current literature. There is a pressing need for more research that includes large numbers of autistic girls/women in order to better understand their physical health. This should be prioritized in order to advance the best clinical care for autistic individuals [11].

Emerging patterns of co-occurring physical health conditions are worth further examination and replication. With respect to Theme 1 , the current literature suggests that autistic girls/women tend to have more overall physical health challenges and lower overall health and quality of life than do autistic boys/men [19-31]. However, apart from epilepsy, it is still unclear which specific conditions are consistently more prevalent in autistic girls/women compared to autistic boys/men or to non-autistic girls/ women [29-31]. Such inconsistency could be related to the substantial heterogeneity in the autism population even within each sex [59] and could also be related to confounding factors (e.g., genetic etiological load or other neurodevelopmental disabilities). Based on Theme 2 , epilepsy is the most studied neurological condition in autism and has been relatively consistently reported to be more prevalent in autistic girls/women than in autistic boys/men [22, 33-35]. Furthermore, these studies highlight heightened autism symptoms, language impairment, motor dysfunction, older age, and presence of intellectual disability as potential confounding factors to epilepsy prevalence in autism [22, 33-35].

Findings in Themes 1 and 2 regarding differences between autistic girls/women and autistic boys/men need to be interpreted in light of the general pattern of case ascertainment in autism research so far, and the complexity associated with sex/gender-related differences in comorbidity pattern (e.g., association with intellectual disability) and etiological load. With regard to ascertainment bias, some have suggested that the observed association between autism and epilepsy is largely driven by co-occurring intellectual disability [60], which is in turn confounded by the greater proportion of autistic girls/ women with lower cognitive abilities [61] and shared neurobiological bases between autism and neurological anomalies [62]. Indeed, for a long time, girls/women who were clinically diagnosed with autism, and hence included in the registry and hospital-based datasets in the reviewed studies, tended to be those with lower IQ, early-recognized and more "classic" symptoms of autism [63]-those who tend to carry heightened etiological risk factors for neurodevelopmental and physical health issues. Meanwhile, sex- and gender-related barriers to girls/women receiving an autism diagnosis $[2,4,11]$ may result in autistic girls/women without evident intellectual disability or "classic" symptoms of autism being under-represented in the current literature. Therefore, the observed differences between autistic boys/men and autistic girls/women in overall physical health status and epilepsy are likely confounded by sex- and gender-based ascertainment bias. It is also likely that the later recognition of autism in some girls/women has resulted in inadequate health care [64-66], further contributing to poorer overall health status. However, it remains unclear if the same pattern of male-female differences holds in autistic individuals who are so far under-recognized and undiagnosed.

At the same time, these observed sex/gender differences could be associated with variations in etiological load. At a group level, autistic girls/women tend to carry more de novo protein-truncating genetic variants likely causal to autism compared to autistic boys/men [67]. Given the pleiotropic effects of many autism-related genes, autistic individuals who carry these variants are more likely to experience other neurodevelopmental (e.g., intellectual disability) and physical health challenges (e.g., epilepsy, other neurological anomalies, cardiovascular defects, obesity) [8]. This means that the observed sex/gender differences may further reflect, and are confounded by, higher co-occurrence of neurodevelopmental disabilities and stronger de novo genetic etiological load in diagnosed autistic girls/women compared to autistic boys/men.

Another pattern that emerged (Theme 3) was the greater burden of co-occurring endocrine or reproductive health concerns in autistic girls/women (e.g., menstruation-related challenges, hormonal conditions, and ovarian cancer). Nevertheless, these findings should be viewed as preliminary, owing to the moderate sample sizes [40-42] and the reliance on self-report questionnaires-rather than direct clinical 
assessments - to characterize female-specific endocrine conditions [38-42]. Interestingly though, this theme can be hypothesis generating with implications for plausible biological mechanisms underlying autism, endocrine and other associated biological alternations. Some have speculated that endocrine dysregulation in autistic girls/ women is partly indicative of altered prenatal sex steroid exposure $[68,69]$, which may contribute to both endocrine dysregulation and autism-related neurodevelopmental and behavioral characteristics [70-72]; see recent emerging empirical support [73-77]. How such prenatal endocrine factors contribute to the mechanisms leading to autism and sex differences in concurrent physical health disorders remains unclear and is an area requiring more in-depth mechanistic investigation. There is growing evidence supporting the role of multidirectional interactions between hormonal regulation, prenatal immune activation, epigenetic regulation in key brain regions, and postnatal environments in producing a range of distinct but related autistic phenotypes [78-80]. It is possible that there are shared mechanisms underlying autism and co-occurring endocrine and immune alterations, with sex differential mechanisms involved.

Finally, there is increasing evidence that gastrointestinal [81] and metabolic/nutritional conditions [82], including obesity and diabetes [83], have a high frequency of co-occurrence in autistic individuals. These conditions could involve shared etiological mechanisms with autism as well as with life experiences (e.g., lifestyle, health care support, medication use) of autistic people. The emerging but preliminary findings regarding sex/ gender differences in these domains suggest that they may be especially important to the physical health of autistic girls/women. Elucidating associated sex/genderrelated mechanisms requires more in-depth research.

\section{Clinical Implications (also see Fig. 2)}

The finding that autistic girls/women experience more overall physical health challenges than non-autistic girls/ women and autistic boys/men is of immediate clinical relevance. Improving physical health is integral to the care of all autistic individuals $[84,85]$. Frontline and

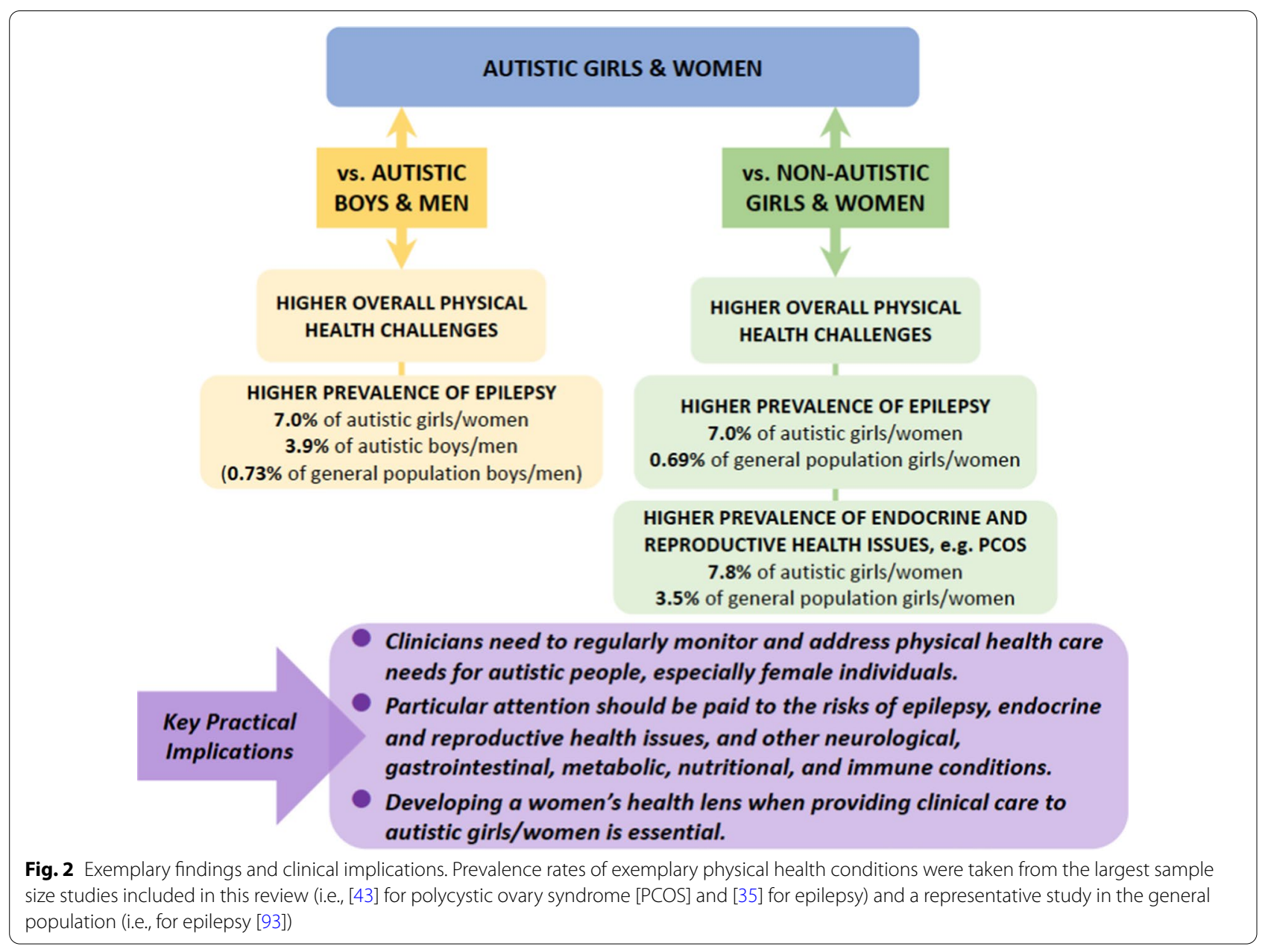


primary care clinicians should regularly monitor and address health care needs for autistic children, youth, and adults alike [86, 87], especially for female individuals [88]. For autistic girls/women, this scoping review provides indication for clinicians to particularly attend to the risks of epilepsy, endocrine and reproductive health issues, as well as other neurological, gastrointestinal, metabolic, nutritional, and immune conditions, among various physical health issues. To achieve holistic support, developing a women's health lens when providing clinical care to autistic girls/women is essential and will significantly enrich sex- and gender-informed health care for all autistic people. Sex- and gender-informed health promotion strategies need to be applied across the life span. Conversely, improved attention to physical health in girls/women who also experience difficulties in social communication, restricted/stereotyped behaviors and sensory issues might also facilitate the identification of later recognized autism in girls/women [89].

Another key consideration is the interplay between physical and mental health. Autistic people are prone to experiencing mental health challenges (which we did not review here) [15]. However, many psychiatric medications for such challenges have side effects that are more commonly experienced in autistic compared to non-autistic individuals [84, 90, 91], contributing to heightened risk to physical health (e.g., weight gain and endocrine problems related to psychotropic medications). These findings have not yet been sufficiently studied in a sex-/gender-specific manner. Meanwhile, physical health challenges (e.g., epilepsy, hormonal dysregulation) can have detrimental impacts on mental health and affect mood and behavior. Such complexity and interplay may result in the high clinical needs and multiple service use that are common in the autism population, particularly among girls/women [64-66]. Many of these specific physical health challenges are treatable with the proviso that clinical trials need to disaggregate their data by sex and gender, which is unfortunately still insufficiently done for trials involving autistic people.

Timely diagnosis and treatment will enhance wellbeing associated with both physical and mental health of autistic individuals across the life span. This review has revealed that autistic girls/women are a population with unique health needs. Therefore, it requires us to develop comprehensive services that integrate developmental, mental, and physical health for autistic girls/women.

\section{Limitations}

There are several limitations to consider in interpreting our findings. First, it is possible that we were unable to identify all studies relevant to our guiding questions due to the heterogeneity in how physical health conditions were assessed and reported in the literature. Nevertheless, based on the principles of a scoping review, we have identified potential areas in the literature that warrant future investigation and areas with insufficient information as yet to make firm conclusions. Second, the decision to focus on physical health, thus excluding studies only focusing on psychiatric co-occurring conditions, meant that we could not explore how mental health and physical health are intertwined in autistic people, particularly in girls/women. Finally, our scoping review demonstrates that understandings of the physical health of autistic girls/women are still emerging. The limited number of studies in each theme, and their varying quality and research methodologies, makes it difficult to reach definitive conclusions.

However, several lessons about significant gaps in the clinical and research literature about autism can be learned from our review to inform future research directions. There is a lack of consistent, basic epidemiological information on the prevalence and incidence for co-occurring physical health conditions in the autism population by sex and, in particular, by gender. Reliable and valid measurement tools for physical health in autistic individuals need to be further developed. Additionally, there are insufficient longitudinal studies to chart the emergence of co-occurring conditions and randomized control trials to assess treatment for these conditions. There are also insufficient biological studies on the mechanisms of the development of physical health challenges in autism by sex and by gender. Further, there is significant underrepresentation of autistic girls/women in most studies, and only a small minority of studies are equipped to or have formally examined and reported sex/gender differences in their primary analyses. These highlight the ignorance about how sex and gender influence autism, perhaps due to a male-biased lens. The relative lack of awareness about women's health and female experiences in the scientific and clinical knowledge about physical health and autism leaves girls/women diagnosed with autism at distinct disadvantages. Targeted research on autistic girls/women is clearly needed. Future studies on autism should be designed to achieve sex/gender equity by ensuring a male/female ratio closer to the general population rate (i.e., 3:1) [4]. For research focusing on delineating sex-related or gender-related effects, balanced inclusion of participants with diverse sexes and genders should be targeted [2, 92]. Finally, it is extremely rare in the current empirical literature that sex (biological attributes) and gender (psychological, social, and cultural attributes) are defined and examined separately and in valid ways. These gaps need to be addressed in future research, alongside a clarification of sources of demographic, clinical, and etiological heterogeneity 
such as age, pubertal stage, developmental trajectories, intellectual functioning, and genetic background. Such clarification is fundamental for future studies to generate etiological and mechanistic insights by studying cooccurring physical health conditions in autism using sex- and gender-informed frameworks.

\section{Conclusions}

To our knowledge, this is the first scoping review on physical health in autistic girls/women. Emerging themes suggest that autistic girls/women tend to have heightened rates of a variety of co-occurring physical health challenges compared to autistic boys/men and non-autistic girls/women. Clinicians should provide holistic care that integrates not only developmental and mental health, but also physical health. Future studies need to include sufficient numbers of autistic girls/women to achieve adequate power, attend to physical health and the intertwined nature of developmental, mental, and physical health, and use sex- and gender-informed lenses.

\section{Supplementary information}

Supplementary information accompanies this paper at https://doi. org/10.1186/s13229-020-00380-z.

Additional file 1. Appendix: Search Strategy.

\section{Abbreviations}

DSM: Diagnostic and Statistical Manual of Mental Disorders; ICD: International Classification of Diseases; OR: Odds ratio; PRISMA: Preferred Reporting Items for Systematic Reviews and Meta-analyses; RR: Risk ratio; SD: Standard deviation; SIR: Standardized incidence ratio.

\section{Acknowledgements}

Not applicable.

\section{Author contributions}

$\mathrm{M}-\mathrm{CL}, \mathrm{GE}$, and $\mathrm{SB}$ conceived and planned the study. CK and SB carried out the literature search, screening, extraction, and summary of data. M-CL and GE supervised the study and contributed to literature screening and summary of findings. $C K, S B, M-C L$, and GE drafted the manuscript. AT, YL, HKB, SHA, and PS contributed to the interpretation of findings and writing of the manuscript. $\mathrm{M}-\mathrm{CL}$ and SHA obtained funding support for the study. CK and SB contributed equally as first authors. M-CL and GE contributed equally as senior authors. All authors read and approved the final manuscript.

\section{Funding}

$\mathrm{M}-\mathrm{CL}$ is supported by a Canadian Institutes of Health Research (CIHR) Sex and Gender Science Chair (GSB 171373), the Innovation Fund of the Alternative Funding Plan for the Academic Health Sciences Centres of Ontario (CAM-18004), the Ontario Brain Institute via the Province of Ontario Neurodevelopmental Disorders (POND) Network (IDS-I I-02), and Women's Xchange. M-CL and SHA are both supported by the O'Brien Scholars Program within the Child and Youth Mental Health Collaborative at the Centre for Addiction and Mental Health and The Hospital for Sick Children, Toronto, the Academic Scholars Award from the Department of Psychiatry, University of Toronto, and the Slaight Family Child and Youth Mental Health Innovation Fund via the Centre for Addiction and Mental Health Foundation. SHA is supported by funding from the US National Institutes of Mental Health, CIHR, and the Cundill Centre for Child and Youth Depression at the Centre for Addiction and Mental Health. AT is supported by the CIHR Post-doctoral Fellowship and the Azrieli Adult
Neurodevelopmental Centre Fellowship. HKB is supported by a Tier 2 Canada Research Chair in Disability \& Reproductive Health, CIHR, and the US National Institutes of Health. YL is supported by CIHR, the US National Institutes of Health, and the Azrieli Foundation. SB is supported by the Ontario Graduate Scholarship and the Department of Sociology, University of Toronto. GE is supported by the Wilfred and Joyce Posluns Chair in Women's Brain Health and Aging, CIHR, Ontario Brain Institute, Alzheimer's Society Canada, and Women's Brain Health Initiative. The funders have no role in the design of the study, the collection, analysis, and interpretation of data, and writing of the manuscript.

\section{Availability of data and materials}

Not applicable.

\section{Ethics approval and consent to participate}

Not applicable.

\section{Consent for publication}

Not applicable.

\section{Competing interests}

The authors declare that they have no competing interests.

\section{Author details}

${ }^{1}$ The Margaret and Wallace McCain Centre for Child, Youth and Family Mental Health, Centre for Addiction and Mental Health, 80 Workman Way, Toronto, ON M6J 1H4, Canada. ${ }^{2}$ Dalla Lana School of Public Health, University of Toronto, Toronto, Canada. ${ }^{3}$ Department of Sociology, University of Toronto, Toronto, Canada. ${ }^{4}$ Azrieli Adult Neurodevelopmental Centre, Centre for Addiction and Mental Health, Toronto, Canada. ${ }^{5}$ Department of Psychiatry, Faculty of Medicine, University of Toronto, Toronto, Canada. ${ }^{6}$ Department of Health and Society, University of Toronto Scarborough, Toronto, Canada. ${ }^{7}$ Department of Psychiatry, The Hospital for Sick Children, Toronto, Canada. ${ }^{8}$ Department of Psychology, University of Toronto, Toronto, Canada. ${ }^{9}$ Autism Research Centre, Department of Psychiatry, University of Cambridge, Cambridge, UK.

${ }^{10}$ Department of Psychiatry, National Taiwan University Hospital and College of Medicine, Taipei, Taiwan. ${ }^{11}$ Tema Genus, Linköping University, Linköping, Sweden. ${ }^{12}$ Rotman Research Institute, Baycrest Hospital, Toronto, Canada.

Received: 27 April 2020 Accepted: 18 September 2020

Published online: 27 October 2020

\section{References}

1. Lyall K, Croen L, Daniels J, Fallin MD, Ladd-Acosta C, Lee BK, et al. The changing epidemiology of autism spectrum disorders. Annu Rev Public Health. 2017;38:81-102.

2. Lai MC, Lombardo MV, Auyeung B, Chakrabarti B, Baron-Cohen S. Sex/ gender differences and autism: setting the scene for future research. J Am Acad Child Adolesc Psychiatry. 2015;54:11-24.

3. Lai MC, Lombardo MV, Baron-Cohen S. Autism. The Lancet. 2014;383:896-910.

4. Loomes R, Hull L, Mandy WPL. What Is the male-to-female ratio in autism spectrum disorder? A systematic review and meta-analysis. J Am Acad Child Adolesc Psychiatry. 2017;56:466-74.

5. Lai MC, Szatmari P. Sex and gender impacts on the behavioural presentation and recognition of autism. Curr Opin Psychiatry. 2020;33:117-23.

6. Whitlock A, Fulton K, Lai MC, Pellicano E, Mandy W. Recognition of girls on the autism spectrum by primary school educators: an experimental study. Autism Res. 2020;13:1358-72.

7. Muskens JB, Velders FP, Staal WG. Medical comorbidities in children and adolescents with autism spectrum disorders and attention deficit hyperactivity disorders: a systematic review. Eur Child Adolesc Psychiatry. 2017;26:1093-103.

8. Vorstman JAS, Parr JR, Moreno-De-Luca D, Anney RJL, Nurnberger Jl, Hallmayer JF. Autism genetics: opportunities and challenges for clinical translation. Nat Rev Genet. 2017;18:362-78.

9. Szatmari P, Georgiades S, Duku E, Bennett TA, Bryson S, Fombonne E, et al. Developmental trajectories of symptom severity and adaptive 
functioning in an inception cohort of preschool children with autism spectrum disorder. JAMA Psychiatry. 2015;72:276-83.

10. Lord C, Bishop S, Anderson D. Developmental trajectories as autism phenotypes. Am J Med Genet. 2015;169:198-208.

11. Mandy W, Lai MC. Towards sex/gender informed autism research. Autism. 2017;21:643-5.

12. Holtmann M, Bolte $S$, Poustka F. Autism spectrum disorders: sex differences in autistic behaviour domains and coexisting psychopathology. Dev Med Child Neurol. 2007:49:361-6.

13. Tsakanikos E, Underwood L, Kravariti E, Bouras N, McCarthy J. Gender differences in co-morbid psychopathology and clinical management in adults with autism spectrum disorders. Res Autism Spectr Disord. 2011;5:803-8.

14. Kreiser NL, White SW. ASD traits and co-occurring psychopathology: the moderating role of gender. J Autism Dev Disord. 2015;45:3932-8.

15. Lai MC, Kassee C, Besney R, Bonato S, Hull L, Mandy W, et al. Prevalence of co-occurring mental health diagnoses in the autism population: a systematic review and meta-analysis. Lancet Psychiatry. 2019;6:819-29.

16. Arksey H, O'Malley L. Scoping studies: towards a methodological framework. Int J Soc Res Methodol Theory Pract. 2005;8:19-32.

17. Tricco A, Lillie E, Zarin W, O'Brien K, Colquhoun H, Levac D, et al. PRISMA extension for scoping reviews (PRISMA-SCR): checklist and explanation. Ann Intern Med. 2018;167:467-73.

18. Moher D, Liberati A, Tetzlaff J, Altman D, The PRISMA Group. Preferred reporting items for systematic reviews and meta-analyses: the PRISMA statement. PLoS Med. 2009;6:e1000097.

19. Rydzewska E, Hughes-McCormack LA, Gillberg C, Henderson A, MacIntyre C, Rintoul J, et al. Prevalence of sensory impairments, physical and intellectual disabilities, and mental health in children and young people with self/proxy-reported autism: observational study of a whole country population. Autism. 2019a;23:1201-9.

20. Rydzewska E, Hughes-Mccormack LA, Gillberg C, Henderson A, Macintyre C, Rintoul J, et al. Prevalence of long-term health conditions in adults with autism: observational study of a whole country population. BMJ Open. 2018:8:e023945-e023945.

21. Rydzewska E, Hughes-McCormack LA, Gillberg C, Henderson A, MacIntyre C, Rintoul J, et al. General health of adults with autism spectrum disorders - a whole country population cross-sectional study. Res Autism Spectr Disord. 2019b;60:59-66.

22. Supekar $K$, Iyer T, Menon V. The influence of sex and age on prevalence rates of comorbid conditions in autism. Autism Res. 2017;10:778-89.

23. Davignon MN, Qian Y, Massolo M, Croen LA. Psychiatric and medical conditions in transition-aged individuals with ASD. Pediatrics. 2018;141(Suppl 4):S335-45.

24. Jones KB, Cottle K, Bakian A, Farley M, Bilder D, Coon H, et al. A description of medical conditions in adults with autism spectrum disorder: a follow-up of the 1980s Utah/UCLA autism epidemiologic study. Autism. 2016;20:551-61.

25. Mason D, McConachie H, Garland D, Petrou A, Rodgers J, Parr JR. Predictors of quality of life for autistic adults. Autism Res. 2018;11:1138-47.

26. Fortuna RJ, Robinson L, Smith TH, Meccarello J, Bullen B, Nobis K, et al. Health conditions and functional status in adults with autism: a crosssectional evaluation. J Gen Intern Med. 2016:31:77-84.

27. Cashin A, Buckley T, Trollor JN, Lennox N. A scoping review of what is known of the physical health of adults with autism spectrum disorder. J Intellect Disabil. 2018;22:96-108.

28. Rubenstein $E$, Wiggins $L D$, Lee $L-C$. A review of the differences in developmental, psychiatric, and medical endophenotypes between males and females with autism spectrum disorder. J Multihandicap Pers. 2015:27:119-39.

29. Cawthorpe D. Comprehensive description of comorbidity for autism spectrum disorder in a general population. Perm J. 2017;21:86-90.

30. Croen LA, Zerbo O, Qian Y, Massolo ML, Rich S, Sidney S, et al. The health status of adults on the autism spectrum. Autism. 2015;19:814-23.

31. Hand BN, Angell AM, Harris L, Carpenter LA. Prevalence of physical and mental health conditions in Medicare-enrolled, autistic older adults. Autism. 2020;24:755-64.

32. Stacy ME, Zablotsky B, Yarger HA, Zimmerman A, Makia B, Lee L-C. Sex differences in co-occurring conditions of children with autism spectrum disorders. Autism. 2014;18:965-74.
33. Amiet C, Gourfinkein I, Bouzamondo A, Tordjman S, Baulac M, Lechat $P$, et al. Epilepsy in autism is associated with intellectual disability and gender: evidence from a meta-analysis. Biol Psychiatry. 2008;64:577-82.

34. Ewen JB, Marvin AR, Law K, Lipkin PH. Epilepsy and autism severity: a study of 6975 children. Autism Res. 2019;12:1251-9.

35. Viscidi EW, Triche EW, Pescosolido MF, McLean RL, Joseph RM, Spence SJ, et al. Clinical characteristics of children with autism spectrum disorder and co-occurring epilepsy. PLoS ONE. 2013;8:e67797-e67797.

36. Bowers K, Wink LK, Pottenger A, McDougle CJ, Erickson C. Phenotypic differences in individuals with autism spectrum disorder born preterm and at term gestation. Autism. 2015;19:758-63.

37. Ben-Itzchak E, Ben-Shachar S, Zachor DA. Specific neurological phenotypes in autism spectrum disorders are associated with sex representation. Autism Res. 2013;6:594-604.

38. Ingudomnukul E, Baron-Cohen S, Wheelwright S, Knickmeyer R. Elevated rates of testosterone-related disorders in women with autism spectrum conditions. Horm Behav. 2007;51:597-604.

39. Pohl A, Cassidy S, Auyeung B, Baron-Cohen S. Uncovering steroidopathy in women with autism: a latent class analysis. Mol Autism. 2014;5:1-12.

40. Hamilton A, Marshal MP, Murray PJ. Autism spectrum disorders and menstruation. J Adolesc Health. 2011;49:443-5.

41. Bitsika V, Sharpley CF. The effects of menarche upon the sensory features of girls with autism spectrum disorder. J Dev Phys Disabil. 2018;30:755-9.

42. Steward R, Crane L, Mairi Roy E, Remington A, Pellicano E. "Life is much more difficult to manage during periods": autistic experiences of menstruation. J Autism Dev Disord. 2018;48:4287-92.

43. Cherskov A, Pohl A, Allison C, Zhang H, Payne RA, Baron-Cohen S. Polycystic ovary syndrome and autism: a test of the prenatal sex steroid theory. Transl Psychiatry. 2018;8:1-10.

44. Chiang HL, Liu CJ, Hu YW, Chen SC, Hu LY, Shen CC, et al. Risk of cancer in children, adolescents, and young adults with autistic disorder. J Pediatr. 2015;166:418-23.

45. Sundelin HEK, Stephansson O, Hultman CM, Ludvigsson JF. Pregnancy outcomes in women with autism: a nationwide population-based cohort study. Clin Epidemiol. 2018;10:1817-26.

46. Memari AH, Ziaee V, Mirfazeli FS, Kordi R. Investigation of autism comorbidities and associations in a school-based community sample. J Child Adolesc Psychiatr Nurs. 2012;25:84-90.

47. Rubenstein E, Schieve L, Wiggins L, Rice C, Van Naarden BK, Christensen $\mathrm{D}$, et al. Trends in documented co-occurring conditions in children with autism spectrum disorder, 2002-2010. Res Dev Disabil. 2018;83:168-78.

48. Mouridsen $\mathrm{SE}$, Rich $\mathrm{B}$, Isager T. Cerebral palsy in individuals with a history of Asperger's syndrome: a Danish nationwide register study based on hospital diagnoses. J Pediatr Neurol. 2013;11:29-34.

49. Broder-Fingert S, Brazauskas K, Lindgren K, lannuzzi D, Van Cleave J. Prevalence of overweight and obesity in a large clinical sample of children with autism. Acad Pediatr. 2014;14:408-14.

50. Garcia-Pastor T, Salinero JJ, Theirs Cl, Ruiz-Vicente D. Obesity status and physical activity level in children and adults with autism spectrum disorders: a pilot study. J Autism Dev Disord. 2019;49:165-72.

51. Yang $X L$, Liang $S$, Zou MY, Sun CH, Han PP, Jiang XT, et al. Are gastrointestinal and sleep problems associated with behavioral symptoms of autism spectrum disorder? Psychiatry Res. 2018:259:229-35.

52. Tseng P, Cheng Y, Chen Y, Stubbs B, Whiteley P, Carvalho A, et al. Peripheral iron levels in children with autism spectrum disorders vs controls: a systematic review and meta-analysis. Nutr Res. 2018;50:44-52.

53. Rossignol DA, Frye RE. Mitochondrial dysfunction in autism spectrum disorders: a systematic review and meta-analysis. Mol Psychiatry. 2012;17:290-314.

54. Guo M, Zhu J, Yang T, Lai X, Lei Y, Chen J, et al. Vitamin A and vitamin D deficiencies exacerbate symptoms in children with autism spectrum disorders. Nutr Neurosci. 2018;22:637-47.

55. Masi A, Breen EJ, Alvares GA, Glozier N, Hickie IB, Hunt A, et al. Cytokine levels and associations with symptom severity in male and female children with autism spectrum disorder. Mol Autism. 2017;8:1-11.

56. $\mathrm{Hu} \mathrm{CC}, \mathrm{Xu} X, \mathrm{Xiong} \mathrm{GL}, \mathrm{Xu} \mathrm{Q}, \mathrm{Zhou} \mathrm{BR}$, Li CY, et al. Alterations in plasma cytokine levels in chinese children with autism spectrum disorder. Autism Res. 2018;11:989-99.

57. Saghazadeh A, Ataeinia B, Keynejad K, Abdolalizadeh A, HirbodMobarakeh A, Rezaei B. A meta-analysis of pro-inflammatory cytokines 
in autism spectrum disorders: effects of age, gender, and latitude. J Psychiatr Res. 2019;115:90-102.

58. Moseley RL, Hitchiner R, Kirkby JA. Self-reported sex differences in high-functioning adults with autism: a meta-analysis. Mol Autism. 2018;9:33-45

59. Lombardo MV, Lai MC, Baron-Cohen S. Big data approaches to decomposing heterogeneity across the autism spectrum. Mol Psychiatry. 2019;24:1435-50.

60. Berg AT, Plioplys S. Epilepsy and autism: is there a special relationship? Epilepsy Behav. 2012;23:193-8.

61. Werling DM, Geschwind DH. Sex differences in autism spectrum disorders. Curr Opin Neurol. 2013;26:146-53.

62. Veliskova J, Silverman JL, Benson M, Lenck-Santini PP. Autistic traits in epilepsy models: why, when and how? Epilepsy Res. 2018;144:62-70.

63. Frazier TW, Georgiades S, Bishop SL, Hardan AY. Behavioral and cognitive characteristics of females and males with autism in the simons simplex collection. J Am Acad Child Adolesc Psychiatry. 2014;53(329-340):e3.

64. Tint A, Weiss JA, Lunsky Y. Identifying the clinical needs and patterns of health service use of adolescent girls and women with autism spectrum disorder. Autism Res. 2017;10:1558-66.

65. Tint A, Weiss JA. A qualitative study of the service experiences of women with autism spectrum disorder. Autism. 2018;22:928-37.

66. Bargiela S, Steward R, Mandy W. The experiences of late-diagnosed women with autism spectrum conditions: an investigation of the female autism phenotype. J Autism Dev Disord. 2016;46:3281-94.

67. Satterstrom FK, Kosmicki JA, Wang J, Breen MS, De Rubeis S, An J-Y, et al. Large-scale exome sequencing study implicates both developmental and functional changes in the neurobiology of autism. Cell. 2020;180(568-584):e23.

68. Rosenfield RL, Ehrmann DA. The pathogenesis of polycystic ovary syndrome (PCOS): the hypothesis of PCOS as functional ovarian hyperandrogenism revisited. Endocr Rev. 2016;37:467-520.

69. Barrett ES, Swan SH. Stress and androgen activity during fetal development. Endocrinology. 2015;156:3435-41.

70. Gore AC, Martien KM, Gagnidze K, Pfaff D. Implications of prenatal steroid perturbations for neurodevelopment, behavior, and autism. Endocr Rev. 2014;35:961-91.

71. Auyeung B, Lombardo MV, Baron-Cohen S. Prenatal and postnatal hormone effects on the human brain and cognition. Pflugers Arch. 2013;465:557-71.

72. Baron-Cohen S, Lombardo MV, Auyeung B, Ashwin E, Chakrabarti B, Knickmeyer R. Why are autism spectrum conditions more prevalent in males? PLoS Biol. 2011;9:e1001081-e1001081.

73. Baron-Cohen S, Tsompanidis A, Auyeung B, Nørgaard-Pedersen B, Hougaard DM, Abdallah M, et al. Foetal oestrogens and autism. Mol Psychiatry. 2019.

74. Baron-Cohen S, Auyeung B, Nørgaard-Pedersen B, Hougaard DM, Abdallah MW, Melgaard L, et al. Elevated fetal steroidogenic activity in autism. Mol Psychiatry. 2015;20:369-76.

75. Kosidou K, Dalman C, Widman L, Arver S, Lee BK, Magnusson C, et al. Maternal polycystic ovary syndrome and the risk of autism spectrum disorders in the offspring: a population-based nationwide study in Sweden. Mol Psychiatry. 2016;21:1441-8.

76. Lee BK, Arver S, Widman L, Gardner RM, Magnusson C, Dalman C, et al. Maternal hirsutism and autism spectrum disorders in offspring. Autism Res. 2017;10:1544-6.
77. Rotem RS, Chodick G, Davidovitch M, Hauser R, Coull BA, Weisskopf MG. Congenital abnormalities of the male reproductive system and risk of autism spectrum disorders. Am J Epidemiol. 2018;187:656-63.

78. Meltzer A, Van De Water J. The role of the immune system in autism spectrum disorder. Neuropsychopharmacology. 2017:42:284-92.

79. Nardone S, Elliott E. The interaction between the immune system and epigenetics in the etiology of autism spectrum disorders. Front Neurosci. 2016;10:329-329.

80. McCarthy MM, Wright CL. Convergence of sex differences and the neuroimmune system in autism spectrum disorder. Biol Psychiatry. 2017:81:402-10.

81. Coury DL, Ashwood P, Fasano A, Fuchs G, Geraghty M, Kaul A, et al. Gastrointestinal conditions in children with autism spectrum disorder: developing a research agenda. Pediatrics. 2012;130(SUPPL. 2):S160-8.

82. Ranjan S, Nasser JA. Nutritional status of individuals with autism spectrum disorders: do we know enough? Adv Nutr. 2015;6:397-407.

83. Shedlock K, Susi A, Gorman GH, Hisle-Gorman E, Erdie-Lalena CR, Nylund CM. Autism spectrum disorders and metabolic complications of obesity. $J$ Pediatr. 2016;178:183-7.

84. Lai MC, Anagnostou E, Wiznitzer M, Allison C, Baron-Cohen S. Evidencebased support for autistic people across the lifespan: maximising potential, minimising barriers, and optimising the person-environment fit. Lancet Neurol. 2020;19:434-51.

85. Nicolaidis C, Raymaker D, McDonald K, Kapp S, Weiner M, Ashkenazy E, et al. The development and evaluation of an online healthcare toolkit for autistic adults and their primary care providers. J Gen Intern Med. 2016:31:1180-9.

86. Karpur A, Lello A, Frazier T, Dixon PJ, Shih AJ. Health disparities among children with autism spectrum disorders: analysis of the national survey of children's health 2016. J Autism Dev Disord. 2019;49:1652-64.

87. Mason D, Ingham B, Urbanowicz A, Michael C, Birtles H, Woodbury-Smith $M$, et al. A systematic review of what barriers and facilitators prevent and enable physical healthcare services access for autistic adults. J Autism Dev Disord. 2019;49:3387-400.

88. Tint A, Hamdani Y, Sawyer A, Desarkar P, Ameis SH, Bardikoff N, et al. Wellness efforts for autistic women. Curr Dev Disord Rep. 2018;5:207-16.

89. Lai MC, Baron-Cohen S. Identifying the lost generation of adults with autism spectrum conditions. Lancet Psychiatry. 2015;2:1013-27.

90. Dove D, Warren Z, McPheeters ML, Taylor JL, Sathe NA, Veenstra-VanderWeele J. Medications for adolescents and young adults with autism spectrum disorders: a systematic review. Pediatrics. 2012;130:717-26.

91. Ameis SH, Kassee C, Corbett-Dick P, Cole L, Dadhwal S, Lai MC, et al. Systematic review and guide to management of core and psychiatric symptoms in youth with autism. Acta Psychiatr Scand. 2018;138:379-400.

92. Strang JF, van der Miesen Al, Caplan R, Hughes C, daVanport S, Lai MC. Both sex- and gender-related factors should be considered in autism research and clinical practice. Autism. 2020;24:539-43.

93. Fiest KM, Sauro KM, Wiebe S, Patten SB, Kwon C-S, Dykeman J, et al. Prevalence and incidence of epilepsy. Neurology. 2017;88:296-303.

\section{Publisher's Note}

Springer Nature remains neutral with regard to jurisdictional claims in published maps and institutional affiliations.

Ready to submit your research? Choose BMC and benefit from:

- fast, convenient online submission

- thorough peer review by experienced researchers in your field

- rapid publication on acceptance

- support for research data, including large and complex data types

- gold Open Access which fosters wider collaboration and increased citations

- maximum visibility for your research: over 100M website views per year

At BMC, research is always in progress.

Learn more biomedcentral.com/submissions 